\title{
CONSTRUCCIÓN Y CARACTERIZACIÓN DE UN DISPOSITIVO EMISOR EN EL INFRARROJO BASADO EN NANOPLAQUETAS DE CARBÓN DE GUADUA ANGUSTIFOLIA KUNTH \\ Construcción y caracterización de un emisor de infrarrojo con nanoplaquetas de carbón vegetal
}

\author{
CONSTRUCTION AND CHARACTERIZATION OF AN INFRARED EMITTING DEVICE BA- \\ SED ON CARBON NANOPLATELETS FROM GUADUA Angustifolia KUNTH \\ Construction and characterization of an infrared emitting based on carbon nanoplatelets
} Leidy Johana Quintero-Rodríguez ${ }^{1}$, J. J. Prías-Barragán² ${ }^{2}$ N. A. Echeverry-Montoya ${ }^{3}$, H. Ariza-Calderón ${ }^{4}$

\author{
${ }^{1 .}$ Ingeniero Electrónico, Universidad del Quindío \\ 2. Instituto Interdisciplinario de las Ciencias, Universidad del Quindío \\ 3. Instituto Interdisciplinario de las Ciencias, Universidad del Quindío \\ 4. Instituto Interdisciplinario de las Ciencias, Universidad del Quindío
}

Recibido: 10 de febrero de 2014
Aceptado: 16 de marzo de 2014
* Correspondencia: Leidy Johana Quintero-Rodríguez, IIC-UQ Cra 15 calle 12N Armenia Q., e-mail: ljquinteror@uqvirtual.edu.co

\begin{abstract}
RESUMEN
En este trabajo presentamos la construcción y la caracterización eléctrica, térmica y óptica de un dispositivo emisor en el infrarrojo (IR) basado en nanoplaquetas de grafito oxidado (GONP) obtenido de la Guadua angustifolia Kunth. Las muestras fueron carbonizadas por pirolisis en ambiente de nitrógeno a $1000 \mathrm{~K}$, pulverizadas por el método de molienda mecánica, seleccionadas en tamizador de $20 \mu \mathrm{m}$ y llevadas a nanopartículas por procesos de cavitación. El tamaño promedio de las nanoplaquetas fue determinado por el análisis de imágenes TEM y AFM. El dispositivo se compone de un arreglo de emisores de IR distribuidos en una matriz. Cada emisor IR fue construido mediante dos placas paralelas de aluminio de forma circular, en el intermedio se encuentra una capa de GONP adherida con pintura de Ag a la placa de aluminio y los dos contactos eléctricos fueron realizados empleando hilo de cobre de $100 \mu \mathrm{m}$ de espesor y pintura de Ag. Las curvas I-V fueron tomadas usando una fuente de corriente de precisión y un nano-voltímetro, encontrándose en la respuesta eléctrica efectos óhmicos y un alto efecto de calentamiento de las placas de aluminio de $10 \mathrm{~K}$ por encima de la temperatura ambiente, lo cual podría atribuirse a la participación fonónica en el transporte eléctrico. Mediante el análisis de imágenes termográficas, se logró determinar el espectro de emisión del dispositivo. Los resultados sugieren que el dispositivo desarrollado, podría ser utilizado como calefactor de bajo consumo energético y emisor de IR para barreras ópticas y comunicaciones ópticas.
\end{abstract}

Palabras claves: Emisor de IR, GONP, Curvas I-V, Imágenes Termográficas. 


\begin{abstract}
We present the construction and the electrical, thermal and optical characterization of an infrared (IR) emitting device based on graphite oxide nanoplatelets (GONP) obtained from Guadua. The samples were carbonized by pyrolysis in nitrogen atmosphere at $1000 \mathrm{~K}$, they were pulverized by mechanical milling, selected on a $20 \mu \mathrm{m}$ sieve, and the resulting powder was turned into nanoparticles by cavitation processes. Nanoplatelets average size was determined by analyzing TEM and AFM images. The device consists of a number of IR emitters arranged in a matrix. Each IR emitter was constructed by two circular parallel aluminum plates, with an intermediate GONP layer adhered to the aluminum plate with silver paint. The two electrical contacts were done using copper wire of $100 \mu \mathrm{m}$ thick and silver paint. The IV curves were taken using a precision current source and a Keithley nanovoltmeter. It was found the electrical response, ohmic effects and ahigh heating effect,observed by an increasing ofthe aluminum plates temperatures of $2,6 \mathrm{~K}$ above room temperature, which could be attributed to the phonon participation in the electricity transport. With the analysis of thermal images it was possible to determine the emission spectrum of the device. The results suggest that the device developed could be used as an energy-efficient heater and an IR emitter for optical communications and optical barriers.
\end{abstract}

Keywords: IR Emitter, GONP, I-V Curves, Thermographic Images.

\section{INTRODUCCIÓN}

El estudio en el campo de la nanotecnología se ha incrementado notablemente en los últimos años debido a las aplicaciones que se pueden desarrollar en los campos de la ingeniería, la medicina, la química, la bioquímica, entre otras [1]. La nanotecnología es considerada como uno de los más innovadores y ambiciosos acontecimientos de la ciencia moderna, como se evidencia en los avances vertiginosos en este campo [1] como, por ejemplo, la comprensión a nivel atómico del efecto de los fármacos, el proceso de creación de fuentes de energía inagotable y el desarrollo de electrónica flexible.

El carbón amorfo o carbón vegetal se caracteriza por no tener una estructura cristalina, aunque puede contener cristales microscópicos de grafito, algunas veces tipo diamante y sus formas alotrópicas pueden llegar a conformar estructuras blandas como el grafito o sustancias más duras como el diamante [2].

El carbón vegetal es un material combustible sólido, frágil, poroso y con un alto contenido en carbono y pequeñas cantidades de oxígeno e hidrogeno que se encuentran unidos químicamente en forma de grupos funcionales, tales como: Carbonilo, carboxilo, fenol, lactona, quinona y grupos éter, entre otros [3]. Es producido por precalentamiento de materiales de tipo vegetal como la Guadua angustifolia Kunth, sometidos a temperaturas hasta los $1000 \mathrm{~K}$, en ausencia de aire [2], [4]. El control se realiza sobre la entrada de aire durante el proceso de pirolisis o de carbonización, para que la materia prima no se queme, como sucede con el fuego convencional, sino que sufra un proceso de descomposición química para formar el carbón vegetal.

Actualmente la búsqueda de nuevos dispositivos se ha encaminado en el uso de materiales de tipo vegetal, específicamente en este trabajo se ha realizado el estudio del comportamiento eléctrico de nanoplaquetas de carbón vegetal (GONP) obtenidas a partir de la Guadua Angustifolia Kunth [5], por su potencial en el desarrollo de dispositivos electrónicos. Los usos específicos se basan en las propiedades electrónicas intrínsecas de cada forma que presenta el carbón [6], [7]. En este sentido, la conductividad térmica y la rigidez mecánica de las nanoplaquetas pueden competir con las del grafito en una gran variedad de aplicaciones en el campo de la micro y nanoelectrónica [8].

\section{MATERIALES Y MÉTODOS}

Se pretende determinar el efecto que presentan las nanoplaquetas de carbón vegetal obtenido de la Guadua Angustifolia Kunth, al ser sometidas a cambios de voltaje y temperatura. Es fundamental la determinación de los valores de voltaje y temperatura para garantizar un comportamiento eléctrico de las nanoplaquetas en este caso como dispositivo emisor de infrarrojo. Para construir el dispositivo se realizó la obtención de las nanoplaquetas de carbón vegetal, la construcción del dispositivo, la caracterización eléctrica en el régimen 
DC y la caracterización óptica.

Obtención de las nanoplaquetas de carbón vegetal a partir del precursor de Guadua Angustifolia Kunth.

Para lograr la carbonización de un material precursor, es necesario realizar la descomposición físico-química bajo la acción del calor y la ausencia de un medio oxidante. Los productos que resultan de la carbonización son gases, líquidos y carbón cuyas cantidades relativas dependen de las propiedades del precursor a tratar y de los parámetros de operación del equipo de calentamiento [9].En la Figura 1se presenta el esquema básico del proceso de carbonización. Este proceso se realiza a temperaturas en el rango comprendido desde $600 \mathrm{~K}$ hasta $1000 \mathrm{~K}$ y tiempos que pueden variar desde dos horas hasta días [10].

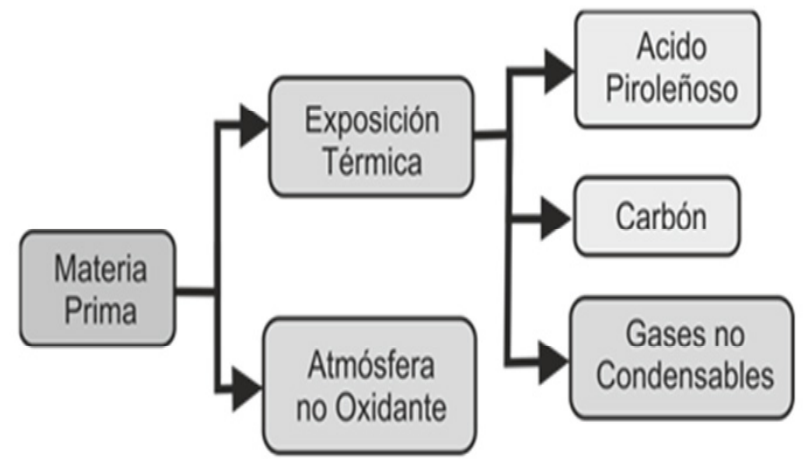

Figura 1. Proceso de carbonización a partir de biomasa

Así, un sistema para obtener carbón de tipo vegetal requiere de una fuente de calor constante, un reactor, el cual puede ser continuo o discontinuo y debe producirse en una atmosfera controlada, libre de oxígeno para evitar que la biomasa se consuma por efectos de la oxidación. Preferiblemente se debe hacer recolección del subproducto de la carbonización mediante la condensación de los vapores resultantes, debido a que los alquitranes y los ácidos piroleñosos pueden tener efectos negativos en la salud y el ambiente.

Las muestras de Guadua fueron cortadas en secciones tipo tabletas de la parte media del culmo de ésta, luego se llevaron a un reactor cilíndrico de pirolisis el cual se encuentra en el interior de una mufla cuya temperatura fue controlada $(1000 \mathrm{~K})$, como se muestra en la Figura 2; al sistema se le realiza un vacío previo para hacer fluir nitrógeno que garantice la mínima cantidad de oxígeno y a partir de la recirculación de agua fría, a través de tres condensadores y un serpentín de vidrio, se produce la condensación de los gases que se generan por la carbonización [11].

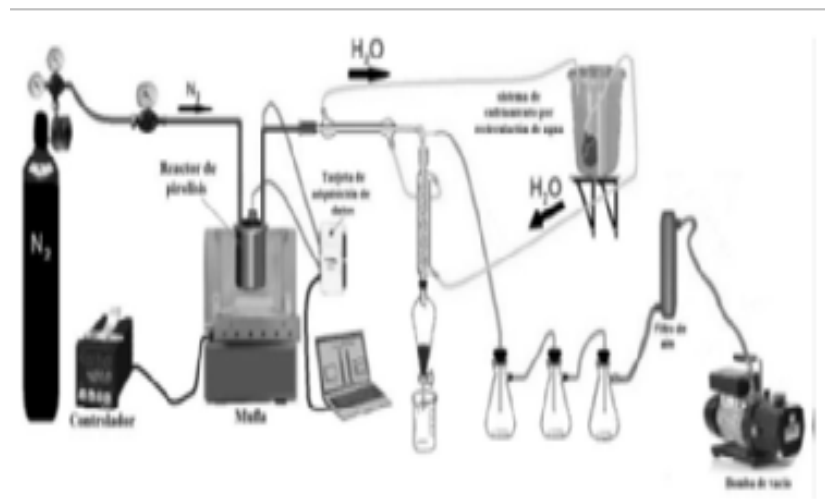

Figura 2. Sistema de pirolisis para la obtención de carbón vegetal a partir del precursor Guadua Angustifolia Kunth [5].

Después de la obtención del carbón de Guadua, se realizó el proceso de molienda de forma manual utilizando un mortero de cerámica hasta obtener un tamaño de grano inferior a $20 \mu \mathrm{m}$, el cual es determinado a través del paso por un tamiz de la marca Ro-Tap Model E, como se muestra en la Figura 3.

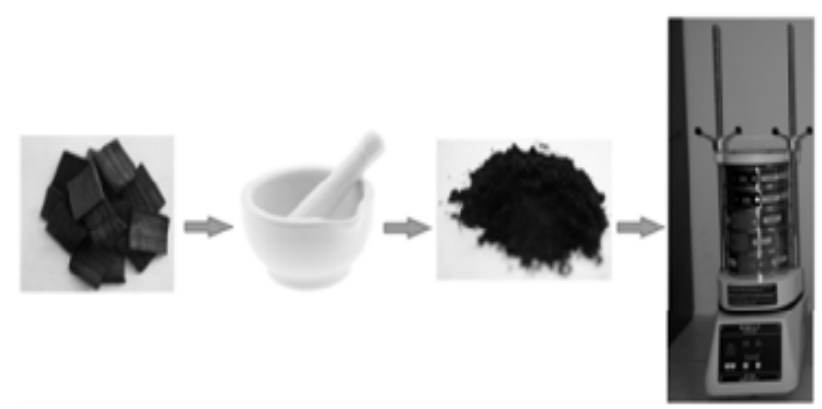

Figura 3. Proceso de maceración y tamizado del carbón vegetal

Las partículas con tamaños de grano menores a 20 $\mu \mathrm{m}$ fueron mezcladas con agua des-ionizada y sometidas a un proceso de cavitación durante seis horas y las nanopartículas, por tener menor peso, fluyen a la superficie y son depositadas en un beacker para ser sometidas a procesos de secado y finalmente obtener las nanopartículas de carbón vegetal.

Construcción del dispositivo emisor de infrarrojos basado en nanoplaquetas de carbón vegetal

En la Figura 4 se presenta la configuración del dis- 
positivo propuesto para la emisión de radiación infrarroja a partir de la obtención de las nanoplaquetas de carbón vegetal. Se puede observar que las GONP se encuentran ubicadas entre las dos placas de aluminio y adheridas con pintura de $\mathrm{Ag}$, mientras que en la Figura 5, se puede observar una imagen de TEM de una GONP. El dispositivo se construyó a partir de dos placas circulares de aluminio, una capa de pintura de plata de alta pureza sobre cada lámina y una capa de las nanoplaquetas de carbón vegetal sobre cada lámina, formando una hetero-estructura. Para evitar que las nanoplaquetas se desplazaran fuera de la superficie, se realizó el sellado del dispositivo utilizando polivinil Alcohol (PVA) en los bordes del dispositivo.

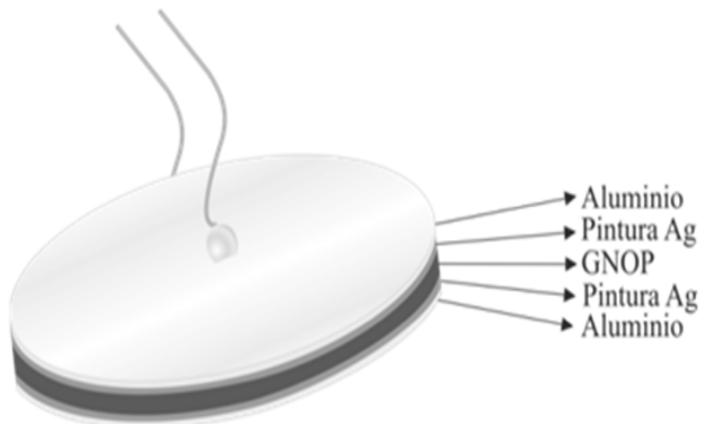

Figura 4 Configuración de dispositivo propuesto de emisor de infrarrojos basado en nanoplaquetas de grafito oxidado (GNOP) [12].

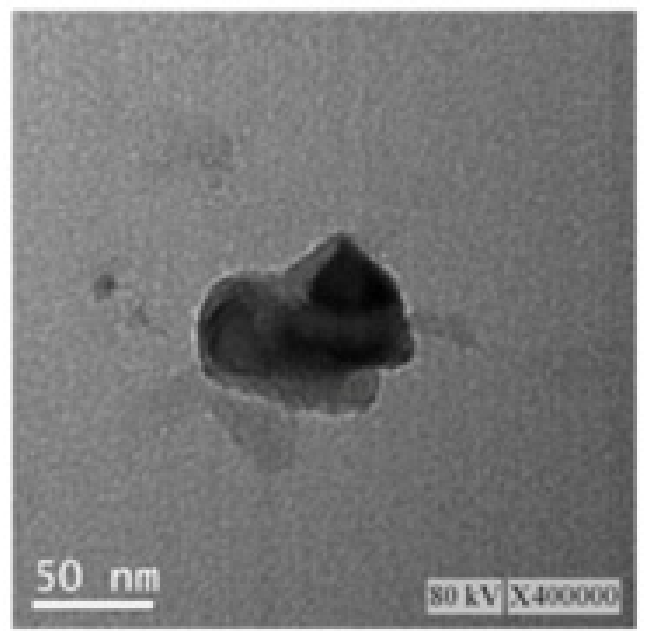

Figura 5. Imagen TEM para las nanoplaquetas de carbón vegetal

\section{Método de dos puntas}

Las medidas de resistencia eléctrica por el método de dos puntas, se realizaron mediante el circuito que se presenta en la Figura 6; se inyecta una corriente I, la cual es común a todos los elementos del circuito conectados en serie ( $\mathrm{R} c$ y $\mathrm{R}$ ). La corriente produce una caída de potencial total $\mathrm{V} m$ que se mide con un multímetro y que es la suma de las caídas de potencial en los cables o sondas Rc, más la de la muestra R. A partir de los valores de I y $\mathrm{V} m$ se calcula el valor de $\mathrm{R}$ con una precisión que dependerá de lo pequeña que sea $\mathrm{R} c$ frente a $\mathrm{R}$.

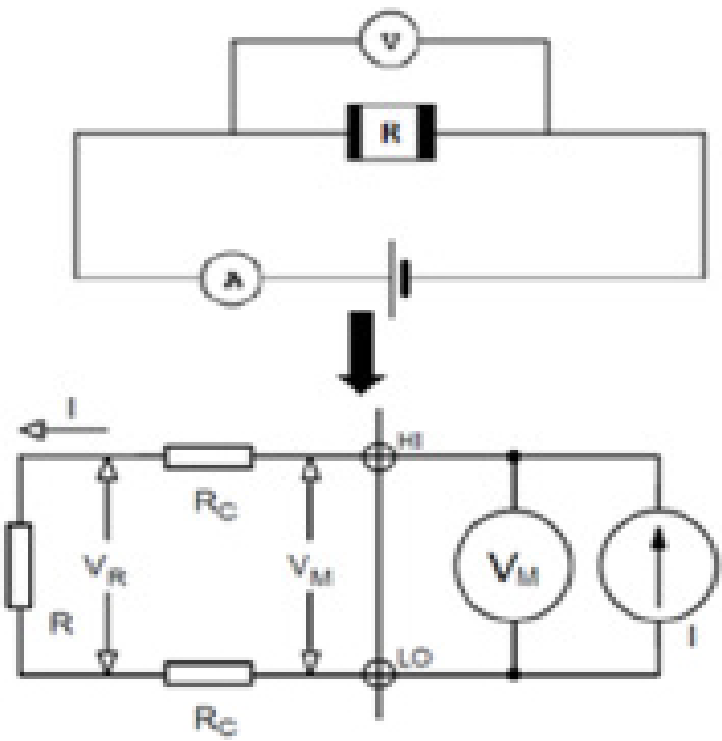

Figura 6. Esquema de medida con el método de dos puntas

Por lo tanto la resistencia medida está dada por la ec. 1:

$$
R_{M}=\frac{V_{M}}{I}=R+2 R_{C}
$$

En donde $\mathrm{R} M$ es la resistencia medida, $\mathrm{Vm}$ es el voltaje medido por el multímetro, $\mathrm{V} R$ es la caída de potencial en la muestra, $\mathrm{R}$ es la resistencia que se quiere medir, $\mathrm{R} c$ es la resistencia de los contactos, e I es la corriente inyectada por la fuente.

Una vez introducida la corriente de excitación, se producen pequeñas caídas de potencial debido a la resistencia de los cables de conexión y a la resistencia de los contactos (de las pinzas con las puntas de prueba y de las puntas de prueba con la muestra). El voltaje medido por el voltímetro $\mathrm{V} M$ nunca será igual que $\mathrm{V} R$, siendo sus valores más próximos en la medida que $\mathrm{R} c<<\mathrm{R}$. Valores típicos de resistencia de los cables que provocan estos errores están en el rango de $1 \mathrm{~m} \Omega$ a $10 \mathrm{~m} \Omega$, por lo que este método está limitado a 
medidas exactas de muestras con resistencia inferiores a $1 \Omega$ o a $10 \Omega[13]$.

\section{Caracterización eléctrica en el régimen DC para el dispositivo emisor de infrarrojo}

Se utilizó el método de las dos puntas considerando el circuito de la Figura 7 en el cual se aplica corriente y se mide voltaje. El transporte eléctrico en el dispositivo puede ser analizado a partir del modelo de Drude Lorentz dado por la ec. 2, la cual predice la difusión de portadores de carga eléctrica en presencia de un campo eléctrico externo.

$$
m_{e} \frac{d v}{d t}=-e E-k v
$$

En donde me es la masa del electrón, $v$ es la velocidad, e es la carga del electrón, E es el campo eléctrico y $k$ es una constante de fuerza de resistencia que obedece a efectos de interacción de los electrones con los fonones.

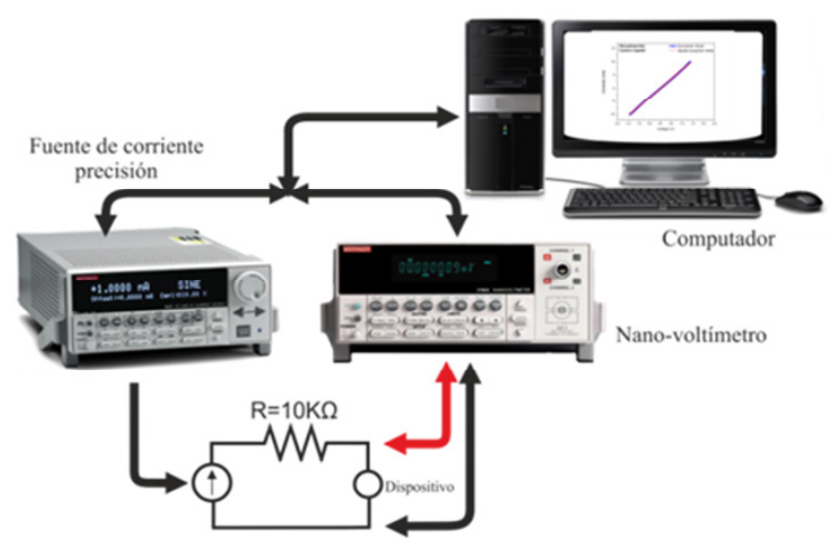

Figura 7. Montaje experimental para la caracterización del dispositivo emisor de infrarrojos basado en nanoplaquetas de grafito oxidado (GNOP) [12]

A partir de la construcción del dispositivo de placas circulares, como lo mencionamos antes, se realizó la caracterización eléctrica en el régimen DC aplicando una variación de corriente y determinando el voltaje en el dispositivo. De las respectivas curvas corrientevoltaje se determinó el valor de la resistencia $\mathrm{R}$ del dispositivo, el cual está relacionado con la conductividad eléctrica, mediante la longitud y el radio , tal como aparece en la expresión [3], la cual puede ser obtenida a partir de la ec. 2 [2].

$$
\sigma=\frac{n e^{2} \tau}{m_{e}}=\frac{\ell}{R S}
$$

Caracterización óptica para el dispositivo emisor de infrarrojo

Se sabe que la cantidad de radiación emitida por un objeto aumenta a medida que aumenta su temperatura. La termografía es un tipo de obtención de imágenes por radiación infrarroja, ya que de acuerdo a la radiación emitida por el objeto cada longitud de onda de esta radiación corresponde a una temperatura diferente. La termografía es el uso de las imágenes infrarrojas con el fin de ver y medir la energía térmica emitida por un objeto.

La termografía electrónica se basa en la detección de temperatura a distancia debido a que el objeto se comporta como un emisor de radiación infrarroja. En este método se utiliza la ecuación de Stephan-Boltzmann con una pequeña variación, debido a que se tiene en cuenta el factor de emisión del cuerpo. Las cámaras termográficas detectan radiación en el espectro electromagnético entre 900 y 14000 nanómetros de longitud de onda y producen imágenes de esta radiación. La imagen que se obtiene de una cámara termográfica es bidimensional en la cual se representa el color irradiado por las diferentes partes del objeto, aunque la distribución de temperatura del objeto es tridimensional. Esta función bidimensional representa la temperatura en función del espacio y la información se almacena en forma matricial [14]. De estas cámaras se obtienen imágenes en escala de grises o de color. En este trabajo se utilizaron las imágenes en escala de grises por facilidad para procesar la imagen y determinar la Transformada dedistribución de potencia de Fourier.

Se realizó la caracterización óptica del dispositivo emisor de infrarrojo emitiendo sobre el dispositivo un haz de luz infrarroja con una longitud de onda de $2500 \mathrm{~nm}$. Inicialmente se realizó la captura de una imagen en escala de gris con una constante de emisividad de 0.9 para el dispositivo sin fuente de alimentación, para evaluar cómo cambia el comportamiento del dispositivo cuando se presenta un cambio de temperatura, lo cual se ilustra en la Figura 8 y Figura 9. 


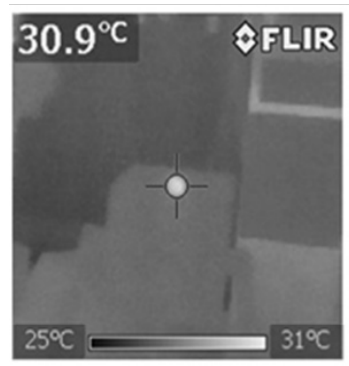

a

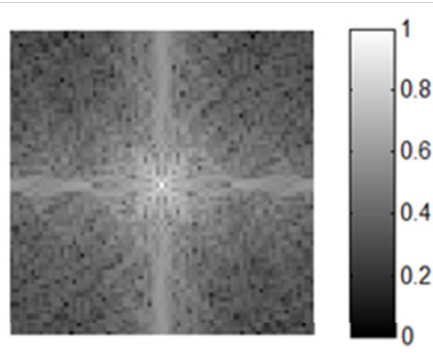

b
Figura 8. Termografía para dispositivo con nanoplaquetas de grafito oxidado (GNOP), sin fuente de voltaje (a) y Espectro de distribución de potencia de Fourier (b)

Asimismo, se realizó la captura de la imagen termográfica en escala de gris para el dispositivo emisor a un voltaje y corriente constante, en donde se observó un incremento de la temperatura de 303 K. En la Figura 9 a se presenta la imagen en escala de gris para cuando el dispositivo está sometido a una fuente de voltaje de $1 \mathrm{~V}$ y en la Figura 9 b, su espectro de distribución de potencia de Fourier.

De la comparación cualitativa entre las imágenes de las Figuras 8 b y 9 b se puede observar la alta homogeneidad en el espectro de Fourier para la Figura 9 b, lo cual podría sugerir una distribución uniforme del calor, cuando el dispositivo es sometido a una polarización fija de $1 \mathrm{~V}$.

Este resultado es muy importante debido a que se trata de un comportamiento esperado para una fuente uniforme de radiación infrarroja, tal como sucede con los actuales dispositivos electrónicos de emisión de infrarrojos [15] y sugiere que el dispositivo propuesto podría ser utilizado como dispositivo emisor de radiación infrarroja.

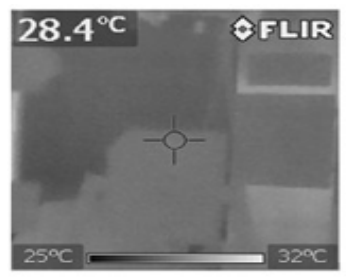

a

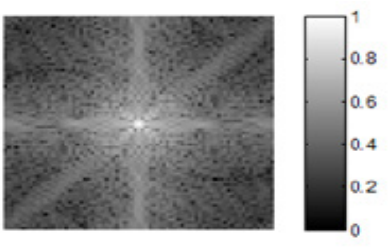

b
Figura 9. Termografía para dispositivo con nanoplaquetas con fuente de voltaje a 1V (a) y Espectro de potencia de Fourier (b)

\section{RESULTADOS Y DISCUSIÓN}

En la Figura 10 se presenta la curva I-V para el dispositivo implementado con GONP. Estos datos experimentales fueron ajustados empleando la ley de Ohm, obteniéndose los valores de $\mathrm{R}$ y usando estos valores en la expresión [3], se determinaron los valores de conductividad eléctrica presentados en la Tabla 1 . Se puede observar en esta tabla que los valores de conductividad eléctrica promedio presentan ordenes de magnitud de 10-3s/m, lo cual concuerda con el orden de magnitud reportado por YanGeng y colaboradores [8] para el caso de nanoplaquetas de grafito oxidado (GONP). Asimismo, se determinó que el valor promedio de la conductividad eléctrica en los dispositivos se encuentra influenciado por el ancho de las plaquetas.

En la Figura 11 se presenta la evolución de la temperatura del dispositivo para valores de voltaje y corriente constante durante cinco minutos, encontrándose que a partir de los primeros 50 segundos se alcanza una temperatura de estado estacionario, tal como era de esperarse, para un dispositivo resistivo con comportamiento térmico de sistema de primer orden. Este efecto de incremento en la temperatura del dispositivo hasta un estado estacionario, podría atribuirse a efectos de calentamiento Joule por la participación fonónica en el transporte eléctrico. El efecto de calentamiento se estudió realizando termografías en el dispositivo para voltaje y corriente constante.

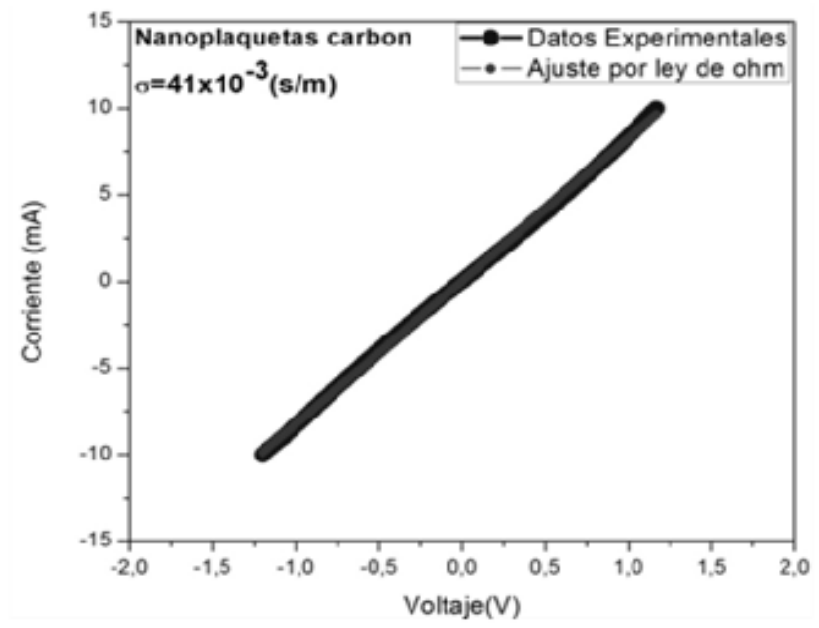

Figura 10. Curva I-V para el dispositivo implementado con nanoplaquetas de grafito oxidado (GNOP) 
Tabla 1. Valores de conductividad eléctrica para el dispositivo con nanoplaquetas de grafito oxidado (GNOP)

\begin{tabular}{|l|l|}
\hline \multicolumn{2}{|l|}{$\begin{array}{l}\text { Conductividad nanoplaquetas (x10-3s/m) valor } \\
\text { promedio 69x10-3 }\end{array}$} \\
\hline $56 \pm 12$ & $0,32 \pm 0,03$ \\
\hline $114 \pm 23$ & $0,29 \pm 0,03$ \\
\hline $41 \pm 8$ & $0,20 \pm 0,02$ \\
\hline $94 \pm 11$ & $0,39 \pm 0,02$ \\
\hline $42 \pm 8$ & $0,29 \pm 0,02$ \\
\hline
\end{tabular}

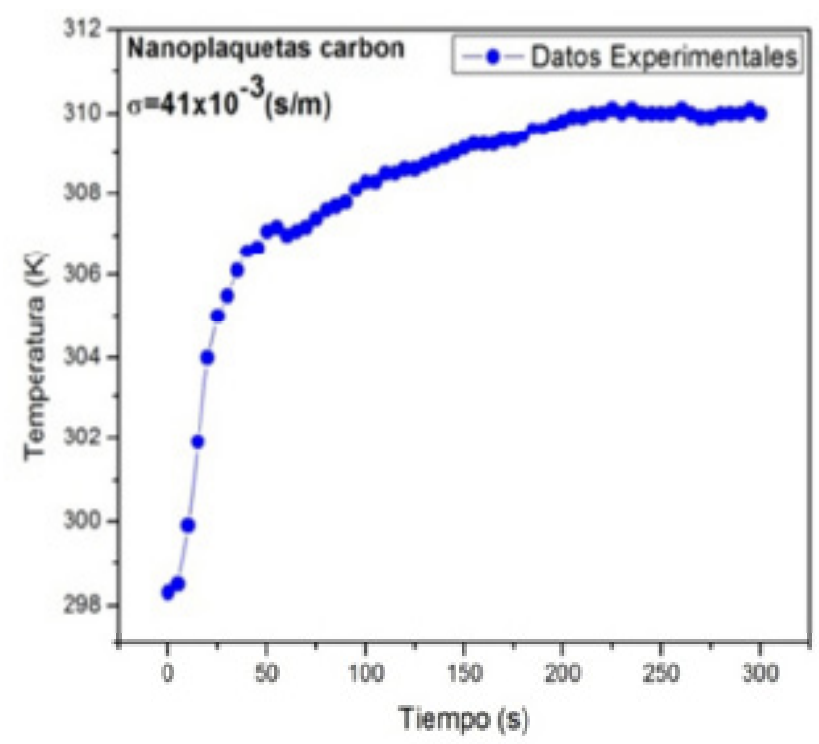

Figura11. Curva Temperatura en función del tiempo para dispositivo con nanoplaquetas de grafito oxidado (GNOP)

A partir de las imágenes termográficas y la transformada de la distribución de potencia de Fourier para cada imagen, se encontró que cuando el dispositivo se encuentra sometido a un valor de voltaje y corriente constante, en el espectro de potencia de Fourier de la Figura 9 b, el comportamiento del espectro de potencia de Fourier es diferente a cuando el dispositivo está sin polarización. Se puede observar en la Figura $9 \mathrm{~b}$ que no aparece la línea diagonal entre las líneas vertical y horizontal que están en una tonalidad de gris claro, tal como aparece en la Figura 8 b, lo cual se podría atribuir a que la temperatura en el dispositivo presenta una distribución homogénea sobre las placas del metal. Para el espectro de la Figura 8 b, la distribución del gris es diferente y se puede observar una línea con un ángulo de $45^{\circ}$ que cruza el eje de la gráfica, que es característica de una imagen que presenta inhomogeneidades en la distribución del calor.

\section{CONCLUSIONES}

Se ha presentado el estudio del comportamiento eléctrico del dispositivo emisor de infrarrojo con nanoplaquetas de carbón vegetal obtenido de la Guadua Angustifolia Kunth, encontrándose que el valor de la conductividad eléctrica concuerda con los reportadosen la literatura especializada para las nanoplaquetas de grafito oxidado.

El efecto de calentamiento tipo Joule observado en las imágenes termográficas sugiere la participación fonónica en el transporte eléctrico.

Se estudió la evolución de la temperatura en el dispositivo bajo la polarización constante de $1 \mathrm{~V}$, observándose un comportamiento característico de sistema de primer orden.

Se propone el dispositivo estudiado para aplicaciones tales como: Calefactores de baja potencia eléctrica, sensores de temperatura, cámaras termográficas y emisores-receptores en el rango espectral del infrarrojo.

\section{AGRADECIMIENTOS}

Al Instituto Interdisciplinario de las Ciencias y a la Universidad del Quindío. 


\section{BIBLIOGRAFÍA}

1. Elizabeth Ayala B, Yeison G. Peña, Oveimar Barbosa, Rodrigo Torres, Fernando Martínez O, Evaluación del efecto térmico de nanotubos de carbono de pared simple funcionalizados con ácido fólico.Armenia: Revista de Investigaciones Universidad del Quindío, 2013. 24.

2. Jiménez, Cotillas Vicente. Sintesis, activación quimica y aplicaciones de nanoestructuras de carbono. Ciudad Real. Universidad de Castilla- La Mancha, 2011.

3. S. Bebelis, H. Karasalis, C. G. Vayenas. Electrochemical promotion of the CO2 hydrogenation on Pd/YSZ and Pd/ ß"'-A12O3 catalyst-electrodes. Solid State Ionics, Vol. 179, 2008.

4. E. I. Papaioannou, S. Souentie, A. Hammad, C. G. Vayenas.Electrochemical promotion of the $\mathrm{CO} 2$ hydrogenation reaction using thin $\mathrm{Rh}, \mathrm{Pt}$ and $\mathrm{Cu}$ films in a monolithic reactor at atmospheric pressure. Catal Today, 2009.

5. J. J. Prías Barragán, Carlos A. Rojas G, N.A. Echeverry M, G. Fhontal, H. Ariza-Calderón. Identificación de las variables óptimas para la obtención de carbón activado a partir del precursor Guadua Angustifolia Kunth.Revista de la Academia Colombiana de Ciencias Exactas, Físicas y Naturales, 2011.

6. M. Alazemi, I. Dutta, F. Wang, R. H. Blunk, A. P. Agelopoulos, Adsorption kinetics and nanostructure development during layer by layer assembly of graphene nanoplatelets and amorphous carbon nanospheres. Carbon 48 (2010) 4063-4073.

7. Xian Jiang, Lawrence T. Drzal. Exploring the potential of exfoliated graphene nanoplatelets as the conductive filler in polymeric nanocomposites for bipolar plates. Journal of Power Sources, 2012.

8. Yan Geng, Shu Jun Wang, Jang-Kyo Kim, Preparation of graphite nanoplatelets and graphene sheets, Journal of Colloid and Interface Science 336 (2009) 592-598.

9. Lesme Jaén, R. Pirólisis de biomasa. Experiencias y aplicaciones. [monografía en Internet]*. CEEFE; (2010). [Acceso 20 de Enero de 2014]. Disponible en: http://www.cengicana.org/ portal/SubOtrasAreas/Cogeneracion/Presentaciones/PirolisisBiomasa.pdf.

10. Márquez Montesino, F. Transformación térmica de la madera. [sede Web]*. Monografias. 2010. [Acceso 12 de Diciembre de 2013]. Disponible en: http://www.monografias.com/trabajos15/transformacion-madera/transformacion-madera.shtml.

11. Alimentación, FAO. Organización de las Naciones Unidas para la Agricultura y la alimentación, Métodos simples para fabricar carbón vegetal. 1983. 41.

12. L. J. Quintero-Rodríguez, J. J. Prías-Barragán, N. A. Echeverry-Montoya y H. Ariza-Calderón. Construcción y Caracterización de Dispositivo Electrónico Basado en Nanoplaquetas de Carbón Obtenido de la Guadua. Armenia: XXV CONGRESO NACIONAL DE FÍSICA, 2013.

13. Peinado Sanchez Alberto. Efecto de la deformación plastica severa en la resistividad eléctrica de una aleación Al-5\% Mg-1,2\%Cr. [Tesis de pregrado]. Madrid: Universidad Carlos III de Madrid. Departamento de Física; 2012.

14. Agudelo Nicolás, Juan Esteban Velez, Natalia Arcila y Carolina Cagua, Radiación Infrarroja y Termografía.Programa de Ingeniería Biomédica, Escuela de Ingeniería de Antioquia (EIA) e Instituto de Ciencias de la Salud (CES), 2010.

15. E. Dupont, M. Byloos, T. Oogarah, M. Buchanan, H.C. Liu, Optimization of quantum-well infrared detectors integrated with light-emitting diodes. Elsevier 47 (2005), 132-134. 